\title{
Codimension bounds for the Noether-Lefschetz components for toric varieties
}

\author{
Ugo Bruzzo ${ }^{1,2,3,4,5} \cdot$ William D. Montoya ${ }^{1,6,7}$
}

Received: 28 May 2020 / Revised: 26 November 2020 / Accepted: 7 February 2021 /

Published online: 30 March 2021

(C) The Author(s) 2021

\begin{abstract}
For a quasi-smooth hypersurface $X$ in a projective simplicial toric variety $\mathbb{P}_{\Sigma}$, the morphism $i^{*}: H^{p}\left(\mathbb{P}_{\Sigma}\right) \rightarrow H^{p}(X)$ induced by the inclusion is injective for $p=\operatorname{dim} X$ and an isomorphism for $p<\operatorname{dim} X-1$. This allows one to define the NoetherLefschetz locus $\mathrm{NL}_{\beta}$ as the locus of quasi-smooth hypersurfaces of degree $\beta$ such that $i^{*}$ acting on the middle algebraic cohomology is not an isomorphism. We prove that, under some assumptions, if $\operatorname{dim} \mathbb{P}_{\Sigma}=2 k+1$ and $k \beta-\beta_{0}=n \eta, n \in \mathbb{N}$, where $\eta$ is the class of a 0 -regular ample divisor, and $\beta_{0}$ is the anticanonical class, every irreducible component $V$ of the Noether-Lefschetz locus quasi-smooth hypersurfaces of degree $\beta$ satisfies the bounds $n+1 \leqslant \operatorname{codim} Z \leqslant h^{k-1, k+1}(X)$.
\end{abstract}

Keywords Noether-Lefschetz components · Codimension · Toric varieties

Mathematics Subject Classification $14 \mathrm{C} 22 \cdot 14 \mathrm{~J} 70 \cdot 14 \mathrm{M} 25$

This research was partly supported by PRIN "Geometria delle Varietà Algebriche" and by GNSAGA-INdAM.

$凶 \quad$ Ugo Bruzzo

bruzzo@sissa.it

William D. Montoya

wmontoya@sissa.it

1 SISSA (Scuola Internazionale Superiore di Studi Avanzati), Via Bonomea 265, 34136 Trieste, Italy

2 Departamento de Matemática, Universidad Federal da Paraíba, Campus I, João Pessoa, PB, Brazil

3 Sezione di Trieste, INFN (Istituto Nazionale di Fisica Nucleare), Trieste, Italy

4 IGAP (Institute for Geometry and Physics), Trieste, Italy

5 Arnold-Regge Center for Algebra, Geometry and Theoretical Physics, Torino, Italy

6 ICTP (International Centre for Theoretical Physics), Strada Costiera, 11, 34151 Trieste, Italy

7 Presently at Universidade Estadual de Campinas, São Paulo, Brazil 


\section{Introduction}

The classical Noether-Lefschetz theory is about the Picard number of surfaces in 3-dimensional projective space. Let $\mathcal{U}_{d} \subset \mathbb{P} H^{0}\left(\mathbb{P}^{3}, \mathcal{O}_{\mathbb{P}^{3}}(d)\right)$ be the locus of smooth surfaces of degree $d$ in $\mathbb{P}^{3}$, with $d \geqslant 4$; then the very general surface in $\mathcal{U}_{d}$ has Picard number 1 (for a historical perspective of the Noether-Lefschetz problem and exhaustive references the reader may consult [3]). Moreover, if $Z$ is a component of the locus in $\mathcal{U}_{d}$ whose points correspond to surfaces with Picard number greater than 1 (the Noether-Lefschetz locus), then

$$
d-3 \leqslant \operatorname{codim}_{u_{d}} Z \leqslant\left(\begin{array}{c}
d-1 \\
3
\end{array}\right) .
$$

This result was generalized in $[5,13]$ to quasi-smooth surfaces in projective simplicial toric threefolds satisfying some conditions. ${ }^{1}$ The purpose of the present paper is to extend these bounds to the case of projective simplicial toric varieties of higher odd dimension, see Theorems 2.1 and 3.1 (when the ambient variety has even dimension the problem is trivial as the middle cohomology of hypersurfaces is controlled by the Lefschetz hyperplane theorem).

This short paper is a natural sequel to [7], where the definition of the NoetherLefschetz locus was extended to simplicial projective toric varieties $\mathbb{P}_{\Sigma}^{2 k+1}$ of arbitrary odd dimension. Given an ample class $\beta$ in $\operatorname{Pic}\left(\mathbb{P}_{\Sigma}^{2 k+1}\right)$, one considers sections $f \in$ $\mathbb{P}\left(H^{0}\left(\mathbb{P}_{\Sigma}^{2 k+1}\right), \mathcal{O}_{\mathbb{P}_{\Sigma}^{2 k+1}}(\beta)\right)$ such that $X_{f}=\{f=0\}$ is a quasi-smooth hypersurface. Let $\left.\mathcal{U}_{\beta} \subset \mathbb{P}\left(H^{0}\left(\mathbb{P}_{\Sigma}^{2 k+1}\right), \mathcal{O}_{\mathbb{P}_{\Sigma}^{2 k+1}}(\beta)\right)\right)$ be the open subset parameterizing quasi-smooth hypersurfaces and let $\pi: \chi_{\beta} \rightarrow \mathcal{U}_{\beta}$ be the tautological family. One considers the local system $\mathcal{H}^{2 k}=R^{2 k} \pi_{\star} \mathbb{C} \otimes \mathcal{O}_{\mathcal{U}_{\beta}}$ over $\mathcal{U}_{\beta}$. The associated flat connection (the GaussManin connection) will be denoted by $\nabla$.

Let $0 \neq \lambda_{f} \in H^{k, k}\left(X_{f}, \mathbb{Q}\right) / i^{*}\left(H^{k, k}\left(\mathbb{P}_{\Sigma}^{2 k+1}, \mathbb{Q}\right)\right)$ and let $U \subset \mathcal{U}_{\beta}$ be a contractible open subset around $f$. Finally, let $\lambda \in \mathcal{H}^{2 k}(U)$ be the section defined by $\lambda_{f}$ and let $\bar{\lambda}$ be its image in $\left(\mathcal{H}^{2 k} / F^{k} \mathcal{H}^{2 k}\right)(U)$, where $F^{k} \mathcal{H}^{2 k}=\mathcal{H}^{2 k, 0} \oplus \mathcal{H}^{2 k-1,1} \oplus \cdots \oplus \mathcal{H}^{k, k}$.

Definition 1.1 (Local Noether-Lefschetz locus) $\mathrm{NL}_{\lambda, U}^{k, \beta}=\left\{G \in U \mid \bar{\lambda}_{G}=0\right\}$.

In this paper we continue the study of the Noether-Lefschetz locus and establish lower and upper bounds for the codimension of its components. In Sect. 2 we obtain the lower bound, which, following the terminology in [3], we call the "explicit Noether-Lefschetz theorem for toric varieties." In Sect. 3, using the Hodge theory for hypersurfaces in complete simplicial toric varieties, and the orbifold structure of the quasi-smooth hyper-surfaces (see [1]), we establish the upper bound, extending the ideas in [5].

\footnotetext{
1 A neat way to define the notion of quasi-smooth hypersurface $X$ in a toric variety $\mathbb{P}_{\Sigma}$ is to regard $\mathbb{P}_{\Sigma}$ as an orbifold: then $X$ is quasi-smooth if and only if it is a sub-orbifold of $\mathbb{P}_{\Sigma}$. Heuristically, $X$ is quasi-smooth if its only singularities are those "inherited" from $\mathbb{P}_{\Sigma}$.
} 


\section{Explicit Noether-Lefschetz theorem in toric varieties}

This section is a natural extension to higher dimensions of the ideas developed in $[5,13]$ for the case of threefolds. To this end there are two points to consider:

1. Let

$$
S=\bigoplus_{\beta \in \mathrm{Cl}\left(\mathbb{P}_{\Sigma}^{2 k+1}\right)} S_{\beta}
$$

be the Cox ring of the toric variety $\mathbb{P}_{\Sigma}^{2 k+1}$ under consideration. In $[5,13]$ the following assumption was made in the case $k=1$. Let $\beta$ and $\eta$ be ample classes in $\operatorname{Pic}\left(\mathbb{P}_{\Sigma}^{3}\right)$, with $\eta$ primitive and 0-regular (in the sense of Castelnuovo regularity), and $\beta-\beta_{0}=n \eta$ for some $n \geqslant 0$, where $\beta_{0}$ is the anticanonical class of $\mathbb{P}_{\Sigma}^{3}$. Then one assumes that the multiplication map $S_{\beta} \otimes S_{n \eta} \rightarrow S_{\beta+n \eta}$ is surjective; this implies that a very general quasi-smooth surface of degree $\beta$ in $\mathbb{P}_{\Sigma}^{3}$ has the same Picard number as $\mathbb{P}_{\Sigma}^{3}$. In the higher dimensional case, if we assume again the surjectivity of the multiplication map, using [1, Theorem 10.13 and Proposition 13.7] and [4, Lemma 3.7], one proves that the primitive cohomology of degree $2 k$ of a very general quasi-smooth hypersurface of degree $\beta$ is zero. Of course we recover the result of [10] when $k=1$.

2. In $[4,13]$ it was also assumed that $H^{1}\left(\mathbb{P}_{\Sigma}^{3}, \mathcal{O}_{\mathbb{P}_{\Sigma}^{3}}(\beta-\eta)\right)=H^{2}\left(\mathbb{P}_{\Sigma}^{3}, \mathcal{O}_{\mathbb{P}_{\Sigma}^{3}}(\beta-2 \eta)\right)$ $=0$, which allowed one to conclude that a certain vector bundle was 1 -regular with respect to $\eta$. Here we assume

$$
H^{q}\left(\mathbb{P}_{\Sigma}^{2 k+1}, \mathcal{O}_{\mathbb{P}_{\Sigma}^{2 k+1}}(\beta-q \eta)\right)=0 \text { for } 1 \leqslant q \leqslant 2 k,
$$

and will prove the same regularity for the analogue of that vector bundle.

The next theorem establishes the lower bound for the codimension of the components of the Noether-Lefschetz locus.

Theorem 2.1 Let $\mathbb{P}_{\Sigma}^{2 k+1}$ be a Gorenstein projective simplicial toric variety, $\eta$ a 0 regular primitive ample Cartier class, and $\beta$ a Cartier class such that $k \beta-\beta_{0}=n \eta$, $n>0$, where $\beta_{0}$ is the anticanonical class of $\mathbb{P}_{\Sigma}^{2 k+1}$. Assume that the multiplication morphism $S_{\beta} \otimes S_{n \eta} \rightarrow S_{\beta+n \eta}$ is surjective, and that $H^{q}\left(\mathbb{P}_{\Sigma}^{2 k+1}, \mathcal{O}_{\mathbb{P}_{\Sigma}^{2 k+1}}(\beta-q \eta)\right)=0$ for $q=1, \ldots, 2 k$; then

$$
\operatorname{codim} Z \geqslant n+1
$$

for every irreducible component $Z$ of the Noether-Lefschetz locus.

Proof The proof is a higher dimensional generalization of that in [5] (which in turn largely mimics the proof of $[9,10]$ for the case of $\left.\mathbb{P}^{3}\right)$, with the modification proposed in [13]. We take a base point free linear system $W$ in $H^{0}\left(\mathbb{P}_{\Sigma}^{2 k+1}, \mathcal{O}_{\mathbb{P}_{\Sigma}^{2 k+1}}(\beta)\right)$ and a complete flag of linear subspaces

$$
W=W_{c} \subset W_{c-1} \subset \cdots \subset W_{1} \subset W_{0}=H^{0}\left(\mathbb{P}_{\Sigma}^{2 k+1}, \mathcal{O}_{\mathbb{P}_{\Sigma}^{2 k+1}}(\beta)\right) .
$$


Let $M_{i}$ be the kernel of the surjective map $W_{i} \otimes \mathcal{O}_{\mathbb{P}_{\Sigma}^{2 k+1}} \rightarrow \mathcal{O}_{\mathbb{P}_{\Sigma}^{2 k+1}}(\beta)$, which is locally free. We have to prove that $M_{0}$ is 1-regular with respect to $\eta$, i.e., that $H^{q}\left(\mathbb{P}_{\Sigma}^{2 k+1}\right.$, $\left.M_{0}((1-q) \eta)\right)=0$ for every positive $q$ (this is the regularity property we hinted at in the introduction). Taking cohomology from

$$
0 \rightarrow M_{0} \rightarrow W_{0} \otimes \mathcal{O}_{\mathbb{P}_{\Sigma}^{2 k+1}} \rightarrow \mathcal{O}_{\mathbb{P}_{\Sigma}^{2 k+1}}(\beta) \rightarrow 0
$$

we get

$$
0 \rightarrow H^{0}\left(M_{0}\right) \rightarrow H^{0}\left(W_{0} \otimes \mathcal{O}_{\mathbb{P}_{\Sigma}^{2 k+1}}\right) \stackrel{\pi}{\rightarrow} H^{0}\left(\mathcal{O}_{\mathbb{P}_{\Sigma}^{2 k+1}}(\beta)\right) \rightarrow H^{1}\left(M_{0}\right) \rightarrow 0
$$

as $\pi$ is surjective, $H^{1}\left(M_{0}\right)=0$. The vanishing of $H^{q}\left(\mathbb{P}_{\Sigma}^{2 k+1}, M_{0}(1-q) \eta\right)=0$ for $1<q \leqslant 2 k+1$ is obtained by induction, tensoring the short exact sequence (2) by $\mathcal{O}_{\mathbb{P}_{\Sigma}^{2 k+1}}((1-q) \eta)$, and considering the segment of the long exact sequence of cohomology

$$
\begin{aligned}
\cdots \rightarrow H^{q-1}\left(\mathcal{O}_{\mathbb{P}_{\Sigma}^{2 k+1}}(\beta-(q-1) \eta)\right) & \rightarrow H^{q}\left(M_{0}(-(q-1) \eta)\right) \\
& \rightarrow H^{q}\left(W_{0} \otimes \mathcal{O}_{\mathbb{P}_{\Sigma}^{2 k+1}}(-(q-1) \eta)\right) \rightarrow \cdots
\end{aligned}
$$

where $H^{q-1}\left(\mathcal{O}_{\mathbb{P}_{\Sigma}^{2 k+1}}(\beta-(q-1) \eta)\right)=0$ by the inductive assumption, while

$$
H^{q}\left(\mathbb{P}_{\Sigma}^{2 k+1}, W_{0} \otimes \mathcal{O}_{\mathbb{P}_{\Sigma}^{2 k+1}}(-(q-1) \eta)\right)=0
$$

as $\eta$ is 0 -regular. The remainder of the proof follows as in $[5,13]$.

In [5] the simplicial toric varieties that satisfy the following condition:

if $\alpha$ is an ample Cartier class, and $\beta$ a nef class, then the multiplication morphism $S_{\alpha} \otimes S_{\beta} \rightarrow S_{\alpha+\beta}$ is surjective

were called Oda varieties. Thus, Oda varieties satisfy the surjectivity requirement in Theorem 2.1.

\section{Upper bound for the codimension of the Noether-Lefschetz components in toric varieties}

The explicit Noether-Lefschetz Theorem provides with a lower bound for the codimension of the Noether-Lefschetz components. Hodge theory in toric varieties will give us the upper bound. For a class $\beta$ as in the previous section, let $f$ be a point in the Noether-Lefschetz locus, let $X_{f}$ be the corresponding hypersurface in $\mathbb{P}_{\Sigma}^{2 k+1}$, and let $\lambda$ be a class as in Definition 1.1. 
Theorem $3.1 \operatorname{codim} Z \leqslant h^{k-1, k+1}\left(X_{f}\right)$ for every irreducible component $Z$ of the Noether-Lefschetz locus $\mathrm{NL}_{\lambda, U}^{k, \beta}$.

This section is devoted to proving this theorem. Classically it is a consequence of Griffiths' transversality, which we want to extend to the context of projective simplicial toric varieties.

Variations of Hodge Structure. The tautological family $\pi: X_{\beta} \subset \mathcal{U}_{\beta} \times \mathbb{P}_{\Sigma} \rightarrow \mathcal{U}_{\beta}$ is of finite type and separated since $\mathcal{X}_{\beta}$ and $\mathcal{U}_{\beta}$ are varieties. By [18, Corollary 5.1] there exists a Zariski open set $\mathcal{U} \subset \mathcal{U}_{\beta}$ such that $\mathcal{X}=\pi^{-1}(\mathcal{U}) \rightarrow \mathcal{U}$ is a locally trivial fibration in the classical topology, i.e., there exists an open cover of $\mathcal{U}$ by contractible open sets such that for every element $U$ of the cover and every point $X_{0} \in U$ we have $X_{\mid U} \simeq \pi^{-1}(U) \simeq U \times X_{0}$, which implies that $X_{u} \simeq X_{0}$ for all $u \in U$ as $C^{\infty}$ orbifolds; moreover, $H^{k}\left(X_{u}\right) \simeq H^{k}\left(X_{0}\right)$. Thanks to the locally trivialization and as quasi-smooth hypersurfaces are orbifolds [1], we can put an orbifold structure on $X=\pi^{-1}(U)$.

The Cartan-Lie formula. For every $k$, let $\mathcal{H}^{k}$ be the complex vector bundle on $\mathcal{U}_{\beta}$ associated to the local system $R^{k} \pi_{*} \mathbb{C}$. Let $\Omega$ be a Zariski $k$-form on the orbifold $X$ such that $\Omega_{u}=\Omega_{\mid X_{u}}$ is closed for every $u \in U$; we can associate with it a local section $\omega$ of the vector bundle $\mathcal{H}^{k}$ by letting

$$
\omega(u)=\left[\Omega_{u}\right] \in H^{k}\left(X_{u}, \mathbb{C}\right)
$$

The following result computes the Gauss-Manin connection $\nabla: \mathcal{H}^{k} \rightarrow \mathcal{H}^{k} \otimes \Omega_{\mathcal{U}}$ in the direction $w$ restricted to $X_{0}$.

Proposition 3.2 (Cartan-Lie formula) If $w \in T_{\mathcal{U}, X_{0}}$ and $v \in \Gamma\left(T_{X_{\mid X}}\right)$ is such that $\phi_{*, x}(v)=w$ for all $x \in X_{0}$, one has

$$
\nabla_{w}(\omega)=\left[\iota_{v}(d \Omega)_{\mid X_{0}}\right]
$$

Proof See [15]; actually the proof goes as in the classical case, see [20, Proposition 9.2.2].

Again we take $U$ a contractible open set trivializing $X_{\mathcal{U} \mid U} \simeq U \times X_{0}$.

Definition 3.3 The period map

$$
\mathcal{P}^{p, k}: \mathcal{U} \rightarrow \operatorname{Grass}\left(b^{p, k}, H^{k}(X, \mathbb{C})\right)
$$

is the map which to $u \in U$ associates the term $F^{p} H^{k}\left(X_{u}, \mathbb{C}\right)$ in the Hodge filtration of $H^{k}\left(X_{u}, \mathbb{C}\right) \simeq H^{k}\left(X_{0}, \mathbb{C}\right)$.

Here $b^{p, k}=\operatorname{dim} F^{p} H^{k}\left(X_{u}, \mathbb{C}\right)$. Note that $\mathcal{P}^{p, k}$ is a map of complex manifolds.

Proposition 3.4 The period map $\mathcal{P}^{p, k}$ is holomorphic. 
Proof For the reader's convenience we sketch here a proof of this result, although it has been actually already proved in [15]. By [12, Theorem 7.9] and the fact that Hodge theorem holds also in the orbifold case ([17,22] and also [14, Section 2.1]) $\mathcal{P}^{p, k}$ is a $C^{\infty}$ map. The rest of the proof follows as in [20, Theorem 10.9], whose strategy is to prove that the $\mathbb{C}$-linear extension of the differential to $T_{u} U \otimes \mathbb{C}$ of $\mathcal{P}^{p, k}$ vanishes on the vectors of type $(0,1)$.

Remark 3.5 There is an intrinsic relation between the differential

$$
d \mathcal{P}_{u}^{p, k}(w): F^{p} H^{k}\left(X_{u}\right) \rightarrow H^{k}\left(X_{0}\right) / F^{p} H^{k}\left(X_{u}\right)
$$

and the covariant derivative $\nabla_{w}: \mathcal{H}^{k} \rightarrow \mathcal{H}^{k}$, namely, given $\sigma \in F^{p} H^{k}\left(X_{u}\right)$, one can construct a local section of $\mathcal{H}^{k}$ over $U$

$$
\begin{aligned}
& \tilde{\sigma}: U \rightarrow H^{k}\left(X_{u}\right), \\
& u^{\prime} \mapsto \tilde{\sigma}\left(u^{\prime}\right) \in F^{p} H\left(X_{u^{\prime}}\right)
\end{aligned}
$$

such that $\tilde{\sigma}(u)=\sigma$. Hence,

$$
d \mathcal{P}_{u}^{p, k}(w)(\sigma)=\nabla_{w} \tilde{\sigma} \bmod F^{p} H^{k}\left(X_{u}\right) .
$$

Remark 3.6 The Hodge decomposition

$$
\mathcal{H}^{k}=\bigoplus_{p+q=k} \mathcal{H}^{p, q}
$$

of the bundle $\mathcal{H}^{k}$ is not holomorphic, but the bundles of the Hodge filtration

$$
F^{p} \mathcal{H}^{k}=\bigoplus_{p=0}^{k} \mathcal{H}^{k-p, p}
$$

are. This can be shown using the period map. Indeed by the very definition of the period map one has (see also [20, Section 10.2.1], for the smooth case)

$$
F^{p} \mathcal{H}^{k} \simeq\left(\mathcal{P}^{p, k}\right)^{*} \mathcal{T}_{p, k},
$$

where $\mathcal{T}_{p, k}$ is the tautological bundle on the Grassmannian Grass $\left(b_{p}, H^{k}\left(X_{u_{0}}, \mathbb{C}\right)\right)$. Thus the bundles $F^{p} \mathcal{H}^{k}$ are indeed holomorphic.

Proposition 3.7 (Griffiths transversality)

$$
\nabla F^{p} \mathcal{H}^{k} \subset F^{p-1} \mathcal{H}^{k}
$$

Proof By the Cartan-Lie formula and the above remark

$$
d \mathcal{P}_{w}^{p, k}(\sigma)=\left[\iota_{v} d \Omega_{\mid X_{0}}\right] \bmod F^{p} H^{k}\left(X_{u}\right)
$$


The fact that $\mathcal{P}^{p, k}$ is holomorphic implies that that $\iota_{v} d \Omega_{\mid X_{0}} \in F^{p} H^{k}\left(X_{u}\right)$ if $v$ is of type $(0,1)$, so that if $v$ is of type $(1,0)$ we get $\iota_{v} d \Omega_{\mid X_{0}} \in F^{p-1} H^{k}\left(X_{u}\right)$.

Theorem 3.8 Each $\mathrm{NL}_{\lambda, U}^{k, \beta} \subset \mathcal{U}$ can be defined locally by $h^{k-1, k+1}$ holomorphic equations, where $h^{k-1, k+1}=\mathrm{rk} F^{k-1} \mathcal{H}^{2 k} / F^{k} \mathcal{H}^{2 k}$.

Proof Once Griffiths transversality has been generalized, the proof goes as in the classical case, see [19, Lemma 3.1] and [21, Section 5.3].

This proves Theorem 3.1.

\section{Examples}

3-dimensional examples where the hypotheses of Theorem 2.1 are satisfied were given in [5]. We give here some higher dimensional examples.

Example 4.1 Examples are provided in every dimension by some weighted projective spaces. We recall here two facts about them. Suitable references are $[2,8,16]$.

Let $\mathbb{P}=\mathbb{P}\left[q_{0}, \ldots, q_{n}\right]$, where $\left(q_{0}, \ldots, q_{n}\right)$ is a minimal set of weights, and set $Q=\sum_{i} q_{i}, \delta=\operatorname{lcm}\left(q_{0}, \ldots, q_{n}\right)$. Then $\mathbb{P}$ is Gorestein if and only if $\delta \mid Q$; if this holds true, then the canonical bundle of $\mathbb{P}$ is $\omega=\mathcal{O}_{\mathbb{P}}\left(-\frac{Q}{\delta} \eta\right)$, where $\eta$ is the ample generator of $\operatorname{Pic}(\mathbb{P})$.

Moreover, we shall need the Bott vanishing theorem, which says that

$$
H^{i}\left(\mathbb{P}, \mathcal{O}_{\mathbb{P}}(k)\right)=0 \text { for all } i \neq 0, n \text { and all } k \in \mathbb{Z}
$$

Let $\mathbb{P}_{\Sigma}^{2 k+1}=\mathbb{P}\left[q_{0}, \ldots, q_{2 k+1}\right]$ with

$$
q_{0}=q_{1}=1, \quad q_{i}=2 \text { for } 2 \leqslant i \leqslant 2 k+1 .
$$

Then $Q=4 k+2$ and $\delta=2$, so that $\omega=\mathcal{O}_{\mathbb{P}_{\Sigma}^{2 k+1}}(-(2 k+1) \eta)$. We check that $\eta$ is 0 regular. In view of (3), we need only to check that $H^{2 k+1}\left(\mathbb{P}_{\Sigma}^{2 k+1}, \mathcal{O}_{\mathbb{P}_{\Sigma}^{2 k+1}}(-2 k \eta)\right)=0$. We have indeed

$$
H^{2 k+1}\left(\mathbb{P}_{\Sigma}^{2 k+1}, \mathcal{O}_{\mathbb{P}_{\Sigma}^{2 k+1}}(-2 k \eta)\right)=H^{0}\left(\mathbb{P}_{\Sigma}^{2 k+1}, \mathcal{O}_{\mathbb{P}_{\Sigma}^{2 k+1}}(-\eta)\right)^{*}=0
$$

Then by [5, Proposition 3.11], $\mathbb{P}_{\Sigma}^{2 k+1}$ is an Oda variety.

For every nonnegative $n$ such that $k \mid(n+1)$ the Cartier class $\beta=((n+1) / k+2) \eta$ is ample and satisfies $k \beta-\beta_{0}=n \eta$. Moreover, the vanishings (1) are automatic by the Bott vanishing (3). So all hypotheses of Theorem 2.1 are satisfied.

Example 4.2 Other examples are provided by some products of projectives spaces. All these varieties are Oda by [11, Theorem 3.10] (which is turn is [11, Corollary 4.2]) and [5, Proposition 3.11]. Take for instance $\mathbb{P}_{\Sigma}^{5}=\mathbb{P}^{4} \times \mathbb{P}^{1}$, and choose $\eta=H_{1}+2 H_{2}$, where $H_{1}$ and $H_{2}$ are the hyperplane classes in $\mathbb{P}^{4}$ and $\mathbb{P}^{1}$. Then $\eta$ is primitive, ample and 0-regular, and for every $n$ odd, $n \geqslant 1$, the class $\beta=\frac{n+5}{2} H_{1}+(n+1) H_{2}$ is ample and satisfies $2 \beta-\beta_{0}=n \eta$. The vanishings (1) hold for $n \geqslant 1$. 
Acknowledgements We thank Paolo Aluffi for useful discussions and the referee for her/his suggestions. The results presented here stemmed from a research project initiated by the first author with Antonella Grassi.

Funding Open access funding provided by Scuola Internazionale Superiore di Studi Avanzati - SISSA within the CRUI-CARE Agreement.

Open Access This article is licensed under a Creative Commons Attribution 4.0 International License, which permits use, sharing, adaptation, distribution and reproduction in any medium or format, as long as you give appropriate credit to the original author(s) and the source, provide a link to the Creative Commons licence, and indicate if changes were made. The images or other third party material in this article are included in the article's Creative Commons licence, unless indicated otherwise in a credit line to the material. If material is not included in the article's Creative Commons licence and your intended use is not permitted by statutory regulation or exceeds the permitted use, you will need to obtain permission directly from the copyright holder. To view a copy of this licence, visit http://creativecommons.org/licenses/by/4.0/.

\section{References}

1. Batyrev, V.V., Cox, D.A.: On the Hodge structure of projective hypersurfaces in toric varieties. Duke Math. J. 75(2), 293-338 (1994)

2. Beltrametti, M., Robbiano, L.: Introduction to the theory of weighted projective spaces. Exposition. Math. 4(2), 111-162 (1986)

3. Brevik, J., Nollet, S.: Developments in Noether-Lefschetz theory. In: Doran, R.S., et al. (eds.) Hodge Theory, Complex Geometry, and Representation Theory. Contemporary Mathematics, vol. 608, pp. 21-50. American Mathematical Society, Providence (2014)

4. Bruzzo, U., Grassi, A.: Picard group of hypersurfaces in toric 3-folds. Internat. J. Math. 23(2), \# 1250028 (2012)

5. Bruzzo, U., Grassi, A.: The Noether-Lefschetz locus of surfaces in toric threefolds. Commun. Contemp. Math. 20(5), \# 1750070 (2018)

6. Bruzzo, U., Grassi, A.: On the Hodge conjecture for hypersurfaces in toric varieties. Comm. Anal. Geom. 28(8), 1773-1786 (2020)

7. Bruzzo, U., Montoya, W.D.: An asymptotic description of the Noether-Lefschetz components in toric varieties (2019). arXiv:1905.01570

8. Dolgachev, I.: Weighted projective varieties. In: Carrell, J.B. (ed.) Group Actions and Vector Fields. Lecture Notes in Mathematics, vol. 956, pp. 34-71. Springer, Berlin (1982)

9. Green, M.L.: Koszul cohomology and the geometry of projective varieties. II. J. Differential Geom. 20(1), 279-289 (1984)

10. Green, M.L.: A new proof of the explicit Noether-Lefschetz theorem. J. Differential Geom. 27(1), 155-159 (1988)

11. Ikeda, A.: Subvarieties of generic hypersurfaces in a nonsingular projective toric variety. Math. Z. 263(4), 923-937 (2009)

12. Kodaira, K.: Complex Manifolds and Deformations of Complex Structures. Classics in Mathematics. Springer, Berlin (2005)

13. Lanza, V., Martino, I.: On the codimension of Noether-Lefschetz loci for toric threefolds. Commun. Contempt. Math. 22(5), \# 1950037 (2020)

14. LeBrun, C.: Edges, orbifolds, and Seiberg-Witten theory. J. Math. Soc. Japan 67(3), 979-1021 (2015)

15. Liu, K.F., Zhuang, X.B.: Deformations of complex orbifolds and the period maps. Sci. China Math. 63(1), 83-100 (2020)

16. Rossi, M., Terracini, L.: Linear algebra and toric data of weighted projective spaces. Rend. Semin. Mat. Univ. Politec. Torino 70(4), 469-495 (2012)

17. Saito, M.: Mixed Hodge modules. Publ. Res. Inst. Math. Sci. 26(2), 221-333 (1990)

18. Verdier, J.-L.: Stratification de Whitney et théorème de Bertini-Sard. Invent. Math. 36, 295-312 (1976)

19. Voisin, C.: Hodge loci. In: Farkas, G., Morrison, I. (eds.) Handbook of Moduli, Vol. III. Advanced Lectures in Mathematics (ALM), vol. 26, pp. 507-546. International Press, Somerville (2013) 
20. Voisin, C.: Hodge Theory and Complex Algebraic Geometry. I. Cambridge Studies in Advanced Mathematics, vol. 76. Cambridge University Press, Cambridge (2002)

21. Voisin, C.: Hodge Theory and Complex Algebraic Geometry II. Cambridge Studies in Advanced Mathematics, vol. 77. Cambridge University Press, Cambridge (2003)

22. Wang, Z.Z., Zaffran, D.: A remark on the hard Lefschetz theorem for Kähler orbifolds. Proc. Amer. Math. Soc. 137(8), 2497-2501 (2009)

Publisher's Note Springer Nature remains neutral with regard to jurisdictional claims in published maps and institutional affiliations. 